\title{
On Holomorphically Subprojective Kählerian Manifolds. - III (*).
}

\author{
Semchi YamaguchI - Tyuzr Adati (Tokyo)
}

Dedicated to Professor S. Tachibana on his 50th birthday

Summary. - In two previous papers $[9,10]$ the present authors have established the differential geometric properties of holomorphically subprojective Kählerian manifolds. The purpose of this paper is to seel the necessary and sufficient conditions in order that a Kählerian manifold be the holomorphically subprojective Kählerian manifold of the first kind, corresponding to $P$. Rachevsly's conditions of a subprojeotive Riemannian manifold.

\section{1. - Introduction.}

Let us consider an $n$ complex dimensional Kählerian manifold $M$. If there exusts a complex coordinate system such that every holomorphically planar curve is given with respect to this system by $n-2$ homogeneous linear equations and one other equation that need not be linear, then $M$ is called a holomorphically subprojective Kählerian manifold. $M(n \geqq 3)$ is holomorphically subprojective if and only if there exists a local complex coordinate system $\left(z^{\lambda}\right)(\lambda, \mu, \ldots=1,2, \ldots, n)$ such that the Christoffel symbol may take the form

$$
\Gamma_{\mu \nu}^{\lambda}=\varrho_{\mu} \delta_{\lambda}^{v}+\varrho_{\nu} \delta_{\mu}^{\lambda}+f_{\mu \nu} z^{\lambda}
$$

where $\varrho_{\lambda}$ and $f_{\mu \lambda}$ are self-conjugate vector and symmetric tensor respectively.

The purpose of this paper is to get the necessary and sufficient conditions in order that a Kählerian manifold be the holomorphically subprojective Kählerian manifold of the first kind, corresponding to P. Rachersk's conditions of a subprojective Riemannian manifold. In $\S 2$ we shall give a short summary of Kählerian manifold with vanishing Bochner curvature tensor and holomorphically subprojective Kählerian manifold of the first kind. We consider some partial differential equations and the integrability conditions (I), (II) and (III) of those in $\S 3$. The conditions (I), (II) and (III) are discussed in $\S 4 \sim \S 6$ and in $\S 7$ we prove the main theorem.

(*) Entrata in Redazione il 10 aprile 1976. 


\section{2. - Preliminaries.}

At first we always agree to adopt the summation convention and the following convention: (ij) (resp. [ij]) for indices $i$ and $j$ means the symmetric (resp. skewsymmetric) part with respect to indices $i$ and $j$, for example

$$
\begin{aligned}
u_{(i j)}=u_{i j}+u_{j i} \quad\left(\operatorname{resp} . u_{[i j]}=\right. & \left.u_{i j}-u_{j i}\right) \\
& (i, j, \ldots, r, s, \ldots=1,2, \ldots, 2 n) .
\end{aligned}
$$

Let us consider a $2 n$ real dimensional Kählerian manifold with complex structure $J$ and Riemannian metric $g$ which satisfy the following relations

$$
J_{j}{ }^{r} J_{r}{ }^{h}=-\delta_{j}{ }^{h}, \quad g_{j i}=J_{j}{ }^{r} J_{i}^{s} g_{r s}, \quad \nabla_{j} J_{i}{ }^{h}=0,
$$

$\nabla$ being the operator of covariant derivation with respect to Riemannian connection defined by $g$.

We consider the tensor field given by

$$
\begin{aligned}
& K_{k j i i^{n}}{ }^{n}=R_{k j i}{ }^{n}+\frac{1}{2(n+2)}\left(L_{i\lceil\hbar} \delta_{j\rfloor}{ }^{h}+g_{i \downarrow k} L_{j\rfloor}{ }^{h}\right. \\
& \left.-M_{i[k} J_{j]}^{h}-J_{i[k} M_{j]}^{h}+2 M_{k j} J_{i}{ }^{h}+2 J_{k j} M_{i}{ }^{h}\right)
\end{aligned}
$$

so called the Bochner curvature tensor, where we have put

$$
L_{j i}=R_{j i}-\frac{R}{4(n+1)} g_{j i}, \quad M_{j i}=J_{j}{ }^{r} L_{r i}
$$

In $M$ with vanishing Bochner curvature tensor, the following relation which plays important roles in the following sections has been proved by M. Matsumoto [3]:

$$
4(n+1) \nabla_{k} R_{i i}=g_{k(i} \nabla_{i)} R+2 g_{j i} \nabla_{k} R-J_{k(j)} J_{i)} \nabla_{r} R .
$$

In what follows, we remember the properties of holomorphically subprojective Kählerian manifolds. It is known that in order that a $2 n(n \geqq 3)$ real dimensional Kählerian manifold $M$ is holomorphically subprojective, it is necessary and suffcient that there exists a local real coordinate system $\left(x^{i}\right)$ such that the Christoffel symbol of $M$ may take the form

$$
\begin{gathered}
\left\{\begin{array}{c}
h \\
j i
\end{array}\right\}=\varrho_{(i} \delta_{j\rangle}^{h}+\tilde{\varrho}_{(i i} J_{j)}^{h}+f_{j i} x^{h}-f_{j r} J_{i}{ }^{r} \hat{x}^{h}, \\
f_{[j, i]}=0, \quad f_{r[j} J_{i]}^{r}=0,
\end{gathered}
$$

where $\varrho_{i}$ and $f_{j i}$ are covariant vector and tensor fields respectively, and we have put $\tilde{x}^{h}=J_{r}{ }^{k} x^{r}$ and $\tilde{\varrho}_{h}=-J_{h}^{r} \underline{Q}_{r}$. 
Although the original definition of the holomorphically subprojective Kählerian manifold of the first kind is characterized by some functions, as a new definition we may take the following

THEOREM A [10]. - A $2 n(n \geqq 3)$ dimensional holomorphically subprojective Kählerian manifold $M$ of the first kind has the following properties (I), (II) and (III):

(I) $M$ has a vanishing Bochner curvature tensor which has the Ricci tensor of the form

$$
R_{j k}=\alpha g_{j k}+\beta\left(\sigma_{j} \sigma_{k}+\tilde{\sigma}_{j} \tilde{\sigma}_{t e}\right),
$$

where $\sigma_{i}$ is a gradient vector, and $\alpha$ and $\beta$ are some functions.

(II) The vector $x^{h}$ in (2.4) is contravariant analytio.

(III) There exist a gradient vector $\varrho_{i}$ and a symmetric tensor $f_{j k}$ such that for some functions $\phi$ and $\varphi$

$$
f_{\mathrm{r}[j} J_{i \mathrm{j}}^{r}=0
$$

and

$$
\nabla_{j} \varrho_{i}=-\varrho_{j} \varrho_{i}+\tilde{\varrho}_{j} \tilde{\varrho}_{i}+f_{j i}+\phi g_{j i}+\varphi\left(\sigma_{j} \sigma_{i}+\tilde{\sigma}_{j} \tilde{\sigma}_{i}\right)
$$

\section{3. - Partial differential equations.}

In the following we seek the necessary and sufficient conditions that a $2 n(n \geq 3)$ dimensional Kählerian manifold $M$ be the holomorphically subprojective Kählerian manifold of the first kind. To investigate these some partial differential equations must be considered in $M$ and need seek the integrability conditions of them.

Let us consider now partial differential equations for a vector $z_{i}$

$$
\nabla_{j} z_{i}=-\varrho_{(i} z_{j)}+\tilde{\varrho}_{(i} \tilde{z}_{j)}-f_{j i} z_{r} \xi^{r}+f_{j s} J_{i}^{s} z_{r} \xi^{r},
$$

where $\varrho_{i}$ is a gradient vector, $f_{i i}$ a symmetric tensor satisfying $f_{r i j} J_{i]}^{r}=0$ and $\xi^{h}$ a certain vector, and we have put $\xi^{n}=J_{r}{ }^{k} \xi^{r}, \tilde{\varrho}_{i}=-J_{i}{ }^{r} \varrho_{r}$ and $\tilde{z}_{i}=-J_{i}{ }^{r} z_{r}$.

As for the integrability conditions of (3.1), we shall consider the following three properties:

(I) A Kählerian manifold $M$ has a vanishing Bochner curvature tensor which has the Ricei tensor of the form

$$
R_{j i}=\alpha g_{j i}+\beta\left(\sigma_{j} \sigma_{i}+\tilde{\sigma}_{j} \tilde{\sigma}_{i}\right)
$$

where $\sigma_{i}$ is a gradient vector, and $\beta$ and $\alpha$ are some functions. 
(II) The vector $\xi^{h}=x \sigma^{h}+\mu \sigma^{h}$ for some functions $x$ and $\mu$ is contravariant analytie, that is, $\xi^{h}$ satisfies $\underset{\xi}{\mathcal{L}} J_{i}^{h}=0$, or, equivalently

$$
\nabla_{i} \xi_{i}=J_{i}^{r} J_{i}{ }^{s} \nabla_{r} \xi_{s}
$$

where $\frac{f}{\xi}$ denotes the operator of Lie derivation with respect to $\xi^{n}$.

(III) There exist a gradient vector $\varrho_{i}$ and a symmetric tensor $f_{j i}$ satisfying $f_{r i 2} J_{j 1}^{r}=0$ and

$$
\nabla_{k} \varrho_{j}=-\varrho_{k} \varrho_{j}+\tilde{\varrho}_{k} \tilde{\varrho}_{j}+f_{k j}+\phi g_{k j}+\varphi\left(\xi_{k} \xi_{j}+\tilde{\xi}_{k} \tilde{\xi}_{j}\right)
$$

where $\phi$ and $\phi$ are some functions.

\section{4. - Condition (I).}

In this section, we try to determine the form of $\nabla_{j} \sigma_{i}$ under the condition (I). Then since we have

$$
R_{i i}=\alpha g_{j i}+\beta\left(\sigma_{j} \sigma_{i}+\tilde{\sigma}_{j} \tilde{\sigma}_{i}\right)
$$

where $\sigma_{i}$ is a gradient vector, we have

$$
R=2 n \alpha+2 \beta|\sigma|^{2}
$$

and applying $\nabla_{x}$ to $(4.1)$ and $(4.2)$ respectively, it follows that

$$
\begin{aligned}
& \nabla_{k} R_{j i}=\alpha_{k} g_{j i}+\beta_{k}\left(\sigma_{j} \sigma_{i}+\tilde{\sigma}_{j} \tilde{\sigma}_{i}\right)+\beta\left(\nabla_{k} \sigma_{i} \sigma_{j}+\sigma_{i} \nabla_{k} \sigma_{j}+\nabla_{k} \tilde{\sigma}_{i} \tilde{\sigma}_{j}+\tilde{\sigma}_{i} \nabla_{k} \tilde{\sigma}_{j}\right), \\
& \nabla_{k} R=2 n \alpha_{k}+2|\sigma|^{2} \beta_{k}+4 \beta \sigma^{r} \nabla_{k i} \sigma_{r} .
\end{aligned}
$$

By contraction (4.3) with $g^{k j}$, we get

$$
\nabla_{i} R=2 \alpha_{i}+2\left(\beta_{r} \sigma^{r} \sigma_{i}+\beta_{r} \tilde{\sigma}^{r} \tilde{\sigma}_{i}\right)+2 \beta\left(\nabla_{i} \sigma_{r} \sigma^{r}+\nabla_{r} \sigma^{r} \sigma_{i}+\tilde{\sigma}^{r} \nabla_{r} \tilde{\sigma}_{i}\right)
$$

On the other hand, by our assumption, that is, $K_{k j i}{ }^{k}=0$, we may use (2.3). By virtue of (4.3) and (4.4), it follows from (2.3) that

$$
\begin{aligned}
2(n+1)\left[\alpha_{k} g_{j i}\right. & \left.+\beta_{k}\left(\sigma_{j} \sigma_{i}+\tilde{\sigma}_{i} \tilde{\sigma}_{i}\right)+\beta\left(\nabla_{k} \sigma_{i} \sigma_{j}+\sigma_{i} \nabla_{k} \sigma_{j}+\nabla_{k} \tilde{\sigma}_{i} \tilde{\sigma}_{j}+\tilde{\sigma}_{i} \nabla_{k} \tilde{\sigma}_{j}\right)\right] \\
& =\left(n \alpha_{i}+|\sigma|^{2} \beta_{i}+2 \beta \sigma^{r} \nabla_{r} \sigma_{i}\right) g_{j k} \\
& +2 g_{j i}\left(n \alpha_{k}+\mid \sigma L^{2} \beta_{k}+2 \beta \sigma^{r} \nabla_{r} \sigma_{k}\right)+J_{k(j}\left(n \tilde{\alpha}_{i)}+|\sigma|^{2} \tilde{\beta}_{i)}+2 \beta_{j} \sigma^{r} \nabla_{|r|} \tilde{\sigma}_{i j}\right)
\end{aligned}
$$


and if we contract (4.6) with $\sigma^{i}, \sigma^{i} \sigma^{j}$ and $\sigma^{i} \sigma^{j} \sigma^{k}$ respectively, then we can find

$$
\begin{aligned}
2(n+1)\left[\alpha_{k} \sigma_{j}+\right. & \left.\beta_{k} \sigma_{j}|\sigma|^{2}+\beta\left(\sigma^{r} \nabla_{k} \sigma_{r} \sigma_{j}+|\sigma|^{2} \nabla_{k} \sigma_{j}+\sigma^{r} \nabla_{k} \tilde{\sigma}_{r} \tilde{\sigma}_{j}\right)\right] \\
& =\left(n \alpha_{r}+|\sigma|^{2} \beta_{r}+2 \beta \sigma^{s} \nabla_{r} \sigma_{s}\right) \sigma^{r} g_{k j}+\sigma_{k}\left(n \alpha_{j}+|\sigma|^{2} \beta_{j}+2 \beta \sigma^{r} \nabla_{j} \sigma_{r}\right) \\
& +2 \sigma_{j}\left(n \alpha_{k}+|\sigma|^{2} \beta_{k}+2 \beta \sigma^{r} \nabla_{k} \sigma_{r}\right)+J_{k j}\left(n \tilde{\alpha}_{i}+|\sigma|^{2} \tilde{\beta}_{i}+2 \beta \sigma^{r} \nabla_{r} \tilde{\sigma}_{i}\right) \sigma^{i} \\
& -\tilde{\sigma}_{k}\left(n \tilde{\alpha}_{j}+|\sigma|^{2} \tilde{\beta}_{j}+2 \beta \sigma^{r} \nabla_{r} \tilde{\sigma}_{j}\right)
\end{aligned}
$$

$$
\begin{gathered}
(n+1)|\sigma|^{2}\left(\alpha_{k}+|\sigma|^{2} \beta_{k}+2 \beta \sigma^{r} \nabla_{k} \sigma_{r}\right) \\
=\sigma_{t k}\left(n \alpha_{j}+|\sigma|^{2} \beta_{j}+2 \beta \sigma^{r} \nabla_{j} \sigma_{r}\right) \sigma^{j}+|\sigma|^{2}\left(n \alpha_{k}+|\sigma|^{2} \beta_{k}+2 \beta \sigma^{r} \nabla_{k} \sigma_{r}\right) \\
-\tilde{\sigma}_{\bar{k}}\left(n \tilde{\alpha}_{r}+\tilde{\beta}_{r}+2 \beta \sigma^{s} \nabla_{s} \tilde{\sigma}_{r}\right) \sigma^{r}, \\
\alpha_{r} \sigma^{r}=\left(|\sigma|^{2} \beta_{r}+2 \beta \sigma^{s} \nabla_{r} \sigma_{s}\right) \sigma^{r} .
\end{gathered}
$$

Moreover, contracting (4.7) (resp. (4.8)) with $J^{k i}$ (resp. $\left.\tilde{\sigma}^{k}\right)$, we find

$$
\begin{gathered}
\alpha_{r} \tilde{\sigma}^{r}=\left(|\sigma|^{2} \beta_{r}+2 \beta \sigma^{s} \nabla_{r} \sigma_{s}\right) \tilde{\sigma}^{r} \\
\alpha_{r} \tilde{\sigma}^{r}=0
\end{gathered}
$$

from which

$$
\left(|\sigma|^{2} \beta_{r}+2 \beta \sigma^{s} \nabla_{r} \sigma_{s}\right) \tilde{\sigma}^{r}=0
$$

and therefore by making use of (4.9), (4.11) and (4.12), the equation (4.7) can be rewritten as follows:

$$
\begin{aligned}
& 2 \sigma_{j}\left[\alpha_{k}+n|\sigma|^{2} \beta_{k}+(n-1) \beta \sigma^{r} \nabla_{k} \sigma_{r}\right]+2(n+1) \beta\left(|\sigma|^{2} \nabla_{k} \sigma_{j}+\sigma^{r} \nabla_{k} \tilde{\sigma}_{r} \tilde{\sigma}_{j}\right) \\
& =(n+1) \alpha_{r} \sigma^{r} g_{k j}+\sigma_{k}\left(n \alpha_{j}+|\sigma|^{2} \beta_{j}+2 \beta \sigma^{r} \nabla_{j} \sigma_{r}\right)-\tilde{\sigma}_{k}\left(n \alpha_{j}+|\sigma|^{2} \tilde{\beta}_{j}+2 \beta \sigma^{r} \nabla_{r} \tilde{\sigma}_{j}\right) .
\end{aligned}
$$

Multiplying $J_{m}{ }^{k} J_{n}{ }^{j}$ to $(4.13)$, it holds that

$$
\begin{aligned}
& 2 \tilde{\sigma}_{n}\left[\tilde{\alpha}_{m}+n|\sigma|^{2} \beta_{m b}-(n-1) \beta J_{m}{ }^{k} \sigma^{r} \nabla_{k} \sigma_{r}\right]+2(n+1) \beta\left(|\sigma|^{2} J_{m}{ }^{k} J_{n}{ }^{j} \nabla_{k} \sigma_{j}+J_{m}^{k} \nabla_{k} \tilde{\sigma}_{r} \sigma^{r} \sigma_{j}\right) \\
& =(n+1) \alpha_{r} \sigma^{r} g_{m n}+\tilde{\sigma}_{m}\left(n \tilde{\alpha}_{n}+|\sigma|^{2} \tilde{\beta}_{n}-2 \beta J_{n}{ }^{j} \nabla_{j} \sigma_{r} \sigma^{r}\right)-\sigma_{m}\left(n \alpha_{n}+|\sigma|^{2} \beta_{n}+2 \beta \sigma^{r} \nabla_{r} \sigma_{n}\right)
\end{aligned}
$$

and consequently, by taking account of (4.13) and the equation above we obtain

$$
\begin{aligned}
\sigma_{j}\left[\alpha_{k}+n|\sigma|^{2} \beta_{k}+(n-1) \beta \sigma^{r} \nabla_{k} \sigma_{r}+(n+1) \beta J_{k}^{s} \nabla_{s} \tilde{\sigma}_{r} \tilde{\sigma}^{r}\right] \\
+\tilde{\sigma}_{j}\left[\tilde{\alpha}_{k}+n|\sigma|^{2} \tilde{\beta}_{k}+(n-1) \beta \sigma^{r} \nabla_{r} \tilde{\sigma}_{k}+(n+1) \beta \sigma^{r} \nabla_{k} \tilde{\sigma}_{r}\right] \\
+(n+1) \beta|\sigma|^{2}\left(\nabla_{k} \sigma_{j}+J_{k}{ }^{r} J_{j}^{s} \nabla_{r} \sigma_{s}\right)=(n+1) \alpha_{r} \sigma^{r} g_{j k} .
\end{aligned}
$$


Hence, let us consider the open subset $M^{\prime}$ in $M$ such that

$$
M^{\prime}=\left\{P \in M: \beta \neq 0 \text { and }|\sigma|^{2} \neq 0 \text { at } P\right\} .
$$

Then $M^{\prime}$ is nonempty. In fact, if $M^{\prime}$ is empty, then $M$ is a manifold of constant holomorphic sectional curvature. Also, we can easily know that a manifold of constant holomorphic sectional curvature is holomorphically subprojective. So we shall always consider the our discussions in $M^{\prime}$ in the following.

Transvecting (4.14) with $\tilde{\sigma}^{k}$ and $\tilde{\sigma}^{k} \sigma^{j}$ respectively and regarding to (4.9) and (4.10), we have

$$
\begin{gathered}
\left(\nabla_{r} \sigma_{s}+J_{r}^{\alpha} J_{s}^{b} \nabla_{a} \sigma_{b}\right) \sigma^{r} \sigma^{s} \tilde{\sigma}_{j}=|\alpha|^{2}\left(\sigma^{r} \nabla_{r} \tilde{\sigma}_{j}-\sigma^{r} \nabla_{j} \tilde{\sigma}\right) \\
|\sigma|^{2}\left(\alpha_{k}+n|\sigma|^{2} \beta_{k}+2 n \beta \sigma^{r} \nabla_{k} \sigma_{r}\right)=(n+1) \alpha_{r} \sigma^{r} \sigma_{k} .
\end{gathered}
$$

By the way, we take the skew-symmetric part with respect to indices $k$ and $j$ in (4.13), then

$$
\begin{aligned}
& {\left[(n+2) \alpha_{[k}+(2 n+1)|\sigma|^{2} \beta_{[k}+2 n \beta \sigma^{r} \nabla_{r} \sigma_{[k}\right] \sigma_{j]}} \\
& =\left[n \tilde{\alpha}_{[k}+|\sigma|^{2} \tilde{\beta}_{[k}+2 \beta \sigma^{r} \nabla_{r} \tilde{\sigma}_{[k}-2(n+1) \beta \sigma^{r} \nabla_{[k} \tilde{\sigma}_{[r]}\right] \tilde{\sigma}_{j 1}
\end{aligned}
$$

which yields that

$$
|\sigma|^{2}\left(\alpha_{t a}+|\sigma|^{2} \beta_{k}\right)=\left(|\sigma|^{2} \sigma_{r} \beta^{r}+\alpha_{r} \sigma^{r}\right) \sigma_{k}+|\sigma|^{2} \beta_{r} \tilde{\sigma}^{r} \tilde{\sigma}_{k t},
$$

where we have contracted (4.17) with $\sigma^{j}$ and made use of (4.12) and (4.16), and subtracting (4.4) from (4.5), it follows that

$$
(n-1) \alpha_{i}+|\sigma|^{2} \beta_{i}+\beta\left(\nabla_{i} \sigma_{r}+J_{i}^{s} J_{r}^{t} \nabla_{s} \sigma_{t}\right) \sigma^{r}=\left(\beta_{r} \sigma^{r}+\beta \nabla_{r} \sigma^{r}\right) \sigma_{i}+\beta_{r} \tilde{\sigma}^{r} \tilde{\sigma}_{i},
$$

which means that

$$
(n-1) \alpha_{r} \sigma^{r}+\beta\left(\nabla_{r} \sigma_{s}+J_{r}^{i} J_{s}^{i} \nabla_{i} \sigma_{j}\right) \sigma^{r} \sigma^{s}=\beta|\sigma|^{2} \nabla_{r} \sigma^{r}
$$

If we take account of (4.15) and (4.19), then we get

$$
\begin{aligned}
(n-1)|\sigma|^{2} \alpha_{i}+|\sigma|^{4} \beta_{i}= & |\sigma|^{2} \beta_{r} \tilde{\sigma}^{r} \tilde{\sigma}_{i} \\
& +\left[|\sigma|^{2}\left(\beta_{r} \sigma^{r}+\beta \nabla_{r} \sigma^{r}\right)-\beta\left(\nabla_{r} \sigma_{s}+J_{r}{ }^{t} J_{s}^{j} \nabla^{r} \sigma_{j}\right) \sigma^{r} \sigma^{s}\right] \sigma_{i},
\end{aligned}
$$

from which, by making use of (4.18) and $(4.20)$

$$
|\sigma|^{2} \alpha_{i}=\alpha_{r} \sigma^{r} \sigma_{i}
$$


and therefore this means from $(4.18)$

$$
|\sigma|^{2} \beta_{r t}=\beta_{r} \sigma^{r} \sigma_{b}+\beta_{r} \tilde{\sigma}^{r} \tilde{\sigma}_{l t}
$$

It follows from (4.16) that

$$
2 \beta|\sigma|^{2} \sigma^{r} \nabla_{r} \sigma_{k}=\left(\alpha_{r} \sigma^{r}-|\sigma|^{2} \beta_{r} \sigma^{r}\right) \sigma_{k}-|\sigma|^{2} \beta_{r} \tilde{\sigma}^{r} \tilde{\sigma}_{k},
$$

or, equivalently

$$
2 \beta|\sigma|^{2} \sigma^{r} \nabla_{r} \tilde{\sigma}_{j}=\left(\alpha_{r} \sigma^{r}-|\sigma|^{2} \beta_{r} \sigma^{r}\right) \tilde{\sigma}_{j}+|\sigma|^{2} \beta_{r} \tilde{\sigma}^{r} \sigma_{j}
$$

and by means of (4.20) and (4.24), from (4.15) we have

$$
2 \beta|\sigma|^{2} \sigma^{r} \nabla_{j} \tilde{\sigma}_{r}=\left[(2 n-1) \alpha_{r} \sigma^{r}-|\sigma|^{2} \beta_{r} \sigma^{r}-2|\sigma|^{2} \beta \nabla_{r} \sigma^{r}\right] \tilde{\sigma}_{j}+|\sigma|^{2} \beta_{r} \tilde{\sigma}^{r} \sigma_{j} .
$$

Finally, substituting (4.25) into (4.14), it holds that

$$
\nabla_{k} \sigma_{j}=a g_{k j}+b_{k} \sigma_{j}+c_{k} \tilde{\sigma}_{j}
$$

or, equivalently

$$
\nabla_{z_{j}} \tilde{\sigma}_{j}=a J_{k j}+b_{k} \tilde{\sigma}_{j}-c_{k} \sigma_{j}
$$

where we have put

$$
\left\{\begin{array}{l}
a=\frac{\alpha_{r} \sigma^{r}}{2 \beta|\sigma|^{2}}, \quad b_{j}=-\frac{1}{2 \beta|\sigma|^{2}}\left(\beta_{r} \sigma^{r} \sigma_{j}+\beta_{r} \tilde{\sigma}^{r} \tilde{\sigma}_{j}\right), \\
c_{j}=-\frac{1}{2 \beta|\sigma|^{4}}\left[|\sigma|^{2} \beta_{r} \tilde{\sigma}^{r} \sigma_{j}+\left(2 n \alpha_{r} \sigma^{r}-|\sigma|^{2} \beta_{r} \sigma^{r}-2|\sigma|^{2} \beta \nabla_{r} \sigma^{r}\right) \tilde{\sigma}_{j}\right] .
\end{array}\right.
$$

In the next place, we calculate some identities under the condition (I) which are necessary for what follows.

Now we put

$$
H_{j i}=-\frac{1}{2(n+1)}\left[\frac{(n+2) \alpha-\beta|\sigma|^{2}}{n+1} g_{j i}+\beta\left(\sigma_{j} \sigma_{i}+\tilde{\sigma}_{j} \tilde{\sigma}_{i}\right)\right],
$$

then we have by the condition (I)

$$
\begin{aligned}
-R_{k j i}{ }^{r} u_{r}=u_{[k} H_{j] i}- & \tilde{u}_{[j} H_{k] r} J_{i}^{r}-2 \tilde{u}_{i} H_{k r} J_{j}^{r} \\
& +\frac{\beta}{2(n+1)}\left[g_{i\lfloor k}\left(\sigma_{j 1} \sigma_{r} u^{r}+\tilde{\sigma}_{j 1} \tilde{\sigma}_{r} u^{r}\right)-J_{i\lceil k}\left(\sigma_{j 1} \tilde{\sigma}_{r} u^{r}-\tilde{\sigma}_{j 1} \sigma_{r} u^{r}\right)\right. \\
& \left.+2 J_{k j}\left(\sigma_{i} \tilde{\sigma}_{r} u^{r}-\tilde{\sigma}_{i} \sigma_{r} u^{r}\right)\right]
\end{aligned}
$$


for any vector field $u^{h}$. Operating $\nabla_{k}$ to (4.29) and taking account of (4.26) and (4.27), we can get

$$
-2(n+1) \nabla_{[k} H_{j 1 i}=\frac{\alpha_{r} \sigma^{r}}{2|\sigma|^{2}}\left(g_{i\lfloor j} \sigma_{k]}+\tilde{\sigma}_{[j} J_{k i i}+2 \tilde{\sigma}_{i} J_{k j}\right)
$$

Let us calculate now $\nabla_{i} a=a_{i}$. We obtain with the aid of (4.22) and (4.28)

$$
\alpha_{i}=2 a \beta \sigma_{i}
$$

which means that

$$
\nabla_{j} \alpha_{i}=2\left(a_{j} \beta \sigma_{i}+a \beta_{j} \sigma_{i}+a \beta \nabla_{j} \sigma_{i}\right)
$$

and take the skew-symmetric part in this, then we get

$$
\beta\left(a_{j} \sigma_{i}-a_{i} \sigma_{j}\right)+a\left(\beta_{j} \sigma_{i}-\beta_{i} \sigma_{j}\right)=0 .
$$

At last, contracting this with $\sigma^{i}$ and according to (4.23), it follows that

$$
a_{j}=\frac{1}{\beta|\sigma|^{2}}\left(\beta a_{r} \sigma^{r} \sigma_{j}-a \beta_{r} \tilde{\sigma}^{r} \tilde{\sigma}_{j}\right)
$$

\section{5. - Condition (II).}

Let us consider a vector field $\xi^{n}$ which is given by

$$
\xi^{n}=\varkappa \sigma^{\hbar}+\mu \tilde{\sigma}^{n}
$$

for some functions, from which we obtain

$$
\sigma^{i}=\frac{x \xi^{i}-\mu \tilde{\xi}^{i}}{x^{2}+\mu^{2}}, \quad \tilde{\sigma}^{i}=\frac{x \tilde{\xi}^{i}+\mu \xi^{i}}{x^{2}+\mu^{2}}
$$

Let $u$ s try to rewrite $a_{j}, b_{j}$ and $c_{j}$ by virtue of (5.2). Then we have

$$
\begin{gathered}
b_{j}=-\frac{1}{2 \beta|\xi|^{2}}\left(\beta^{\prime} \xi_{j}+\beta^{\prime \prime} \xi_{j}\right) \\
c_{j}=\frac{1}{2 \beta|\xi|^{2}}\left[\left\{2 \beta \mu\left(\nabla_{r} \sigma^{r}-2 n a\right)-\beta^{\prime \prime}\right\} \xi_{j}+\left\{2 \beta \varkappa\left(\nabla_{r} \sigma^{r}-2 n a\right)+\beta^{\prime}\right\} \xi_{j}\right] \\
a_{j}=\frac{1}{\beta|\xi|^{2}\left(\varkappa^{2}+\mu^{2}\right)}\left[\left\{x \beta\left(x a^{\prime}-\mu a^{\prime \prime}\right)-\mu a\left(\varkappa \beta^{\prime \prime}+\mu \beta^{\prime}\right)\right\} \xi_{j}\right. \\
\left.-\left\{\mu \beta\left(x a^{\prime}-\mu a^{\prime \prime}\right)+\varkappa a\left(\varkappa \beta^{\prime \prime}+\mu \beta^{\prime}\right)\right\} \xi_{j}\right]
\end{gathered}
$$


where we have put

$$
a^{\prime}=a_{r} \xi^{r}, \quad a^{\prime \prime}=a_{r} \xi^{r}, \quad \beta^{\prime}=\beta_{r} \xi^{r}, \quad \beta^{\prime \prime}=\beta_{r} \tilde{\xi}^{r} .
$$

If we contract (5.5) with $\xi^{j}$, then we have

$$
\beta\left(\mu a^{\prime}+\varkappa a^{\prime \prime}\right)+a\left(\varkappa \beta^{\prime \prime}+\mu \beta^{\prime}\right)=0,
$$

which implies from (5.5) that

$$
a_{j}=\frac{1}{|\xi|^{2}}\left(a^{\prime} \xi_{j}+a^{\prime \prime} \tilde{\xi}_{j}\right)
$$

Furthermore the equations $(4.29) \sim(4.31)$ can be rewritten as follows:

$$
\begin{aligned}
& -R_{k j i}{ }^{r} u_{r}=u_{[k} H_{j] i}-\tilde{u}_{[j} H_{k j r} J_{i}{ }^{r}-2 \tilde{u}_{i} H_{k r} J_{j}^{r} \\
& +\frac{\beta}{2(n+1)\left(k^{2}+\mu^{2}\right)}\left[\left(u^{\prime} \xi_{[j}+u^{\prime \prime} \xi_{[j}\right) g_{k] i}+\left(u^{\prime \prime} \xi_{[j}-u^{\prime} \xi_{[j}\right) J_{k] i}+2\left(u^{\prime \prime} \xi_{i}-u^{\prime} \tilde{\xi}_{i}\right) J_{k j}\right], \\
& H_{i k i}=-\frac{1}{2(n+1)}\left[\frac{(n+2) \alpha-\beta|\sigma|^{2}}{n+1} g_{k i}+\frac{\beta}{x^{2}+\mu^{2}}\left(\xi_{k} \xi_{i}+\xi_{k} \xi_{i}\right)\right], \\
& \nabla_{\lfloor k} H_{j] i}=-\frac{a \beta}{2(n+1)\left(x^{2}+\mu^{2}\right)}\left[\varkappa\left(g_{i j} \xi_{k j}+\tilde{\xi}_{[j} J_{k] i}+2 \tilde{\xi}_{i j} J_{k j}\right)\right. \\
& \left.-\mu\left(g_{i j} \xi_{k]}-\xi_{[j} J_{k j i}-2 \xi_{i} J_{k j}\right)\right],
\end{aligned}
$$

where we put $u^{\prime}=u_{r} \xi^{r}$ and $u^{\prime \prime}=u_{r} \xi^{r}$.

Next, differentiating (5.1) covariantly, it follows form $(4.26),(4.27)$ and (5.2) that

$$
\nabla_{j} \xi_{i}=\operatorname{xag}_{j i}+\mu a J_{j i}+\left(b_{j}+v_{j}\right) \xi_{i}+\left(c_{j}+w_{j}\right) \xi_{i}
$$

or, equivalently

$$
\nabla_{j} \xi_{i}=x a J_{j i}-\mu a g_{j \xi}+\left(b_{j}+v_{j}\right) \tilde{\xi}_{i}-\left(c_{j}+w_{j}\right) \xi_{i}
$$

where we put

$$
v_{j}=\frac{x x_{j}+\mu \mu_{j}}{x^{2}+\mu^{2}}, \quad w_{j}=\frac{x \mu_{j}-\mu \varkappa_{j}}{x^{2}+\mu^{2}}
$$

Hence we assume that $\xi^{h}$ is contavariant analytic. Then we have from the condition (II)

$$
\nabla_{j} \xi_{i}-J_{j}{ }^{r} J_{i}{ }^{s} \nabla_{r} \xi_{s}=\left(b_{j}+v_{j}\right) \xi_{i}+\left(c_{j}+w_{j}\right) \xi_{i}-\left(\tilde{b}_{j}+\tilde{v}_{j}\right) \xi_{i}+\left(\tilde{c}_{j}+\tilde{w}_{j}\right) \xi_{i}=0,
$$

which implies that

$$
b_{i}+\tilde{c}_{j}+v_{j}+\tilde{w}_{j}=0 .
$$


In the next section, we deal with (5.16) under the condition (III) and we can see that (5.16) plays important roles in the proof of main theorem.

\section{6. - Condition (IIT).}

Let us now partial differential equations for vector $z_{i}$

$$
\nabla_{j} z_{i}=-\varrho_{(i} z_{j)}+\tilde{\varrho}_{(i} \tilde{z}_{j)}-f_{j i} z_{r} \xi^{r}+f_{j 8} J_{i}^{s} z_{r} \xi^{r}
$$

where $\varrho_{i}$ is gradient and $f_{j i}$ a symmetric tensor satisfying

$$
f_{r i j} J_{i]}^{r}=0
$$

and $\xi^{h}$ is given by (5.1).

Hereafter, we choose $x$ and $\mu$ in (5.1) as follows:

$$
\varrho^{\prime}-x a=-1, \quad \varrho^{\prime \prime}+\mu a=0,
$$

where we set $\varrho^{\prime}=\varrho_{r} \xi^{r}$ and $\varrho^{\prime \prime}=\varrho_{r} \xi^{r}$.

In the first place, we differentiate (6.1) covariantly. Then

$$
\begin{aligned}
\nabla_{k} \nabla_{j} z_{i}=-\nabla_{k} \varrho_{i} z_{j}-\nabla_{k i} \varrho_{j} z_{i}- & \varrho_{i} \nabla_{k} z_{j}-\varrho_{j} \nabla_{k} z_{i} \\
& +\nabla_{k} \tilde{\varrho}_{i} \tilde{z}_{j}+\nabla_{k} \tilde{\varrho}_{j} \tilde{z}_{i}+\tilde{\varrho}_{i} \nabla_{k} \tilde{z}_{j}+\tilde{\varrho}_{j} \nabla_{k} \tilde{z}_{i} \\
& -\nabla_{k} f_{j i} z_{r} \xi^{r}-f_{j i} \nabla_{k t}\left(z_{r} \xi^{r}\right)+\nabla_{k} f_{j s} J_{i}^{s} z_{r} \xi^{r}+f_{j s} J_{i}^{s} \nabla_{k}\left(z_{r} \xi_{r}\right),
\end{aligned}
$$

from which

$$
\nabla_{[k} \nabla_{i j} z_{i}=z_{[k} H_{j] i}^{\prime}+\tilde{z}_{[j} J_{k i]}^{r} H_{i r}^{\prime}+\tilde{z}_{i} J_{[k}^{r} H_{j] r}^{\prime}-z_{r} \xi^{r} Q_{k j i}+z_{r} \tilde{\xi}_{r} Q_{k j r} J_{i}^{r}
$$

where we set

$$
\begin{gathered}
H_{k j}^{\prime}=\nabla_{k} \varrho_{i}+\varrho_{k} \varrho_{i}-\tilde{\varrho}_{k} \tilde{\varrho}_{i}-f_{k i}, \\
Q_{k j i}=\nabla_{[i k} f_{j] i}-\left(-f_{[j}^{\prime}+b_{[j}+v_{[j}\right) f_{k] i}-\left(f_{i j}^{\prime \prime}+c_{\mathrm{L} j}+w_{[j}\right) f_{k i r} J_{i}{ }^{r}, \\
f_{j}^{\prime}=f_{j r} \xi^{r}, \quad f_{j}^{\prime \prime}=f_{j r} \xi^{r} .
\end{gathered}
$$

By our assumption (III), we may choose $\varrho_{i}$ and $f_{j k}$ such that

$$
H_{j k}=H_{j k}^{\prime},
$$

or, equivalently

$$
\begin{aligned}
\nabla_{k} \varrho_{i}+\varrho_{k} \varrho_{i}-\tilde{\varrho}_{k} \tilde{\varrho}_{i} & -f_{k i} \\
& =-\frac{1}{2(n+1)}\left[\frac{(n+2) \alpha-\beta|\sigma|^{2}}{n+1} g_{k i}+\frac{\beta}{x^{2}+\mu^{2}}\left(\xi_{k} \xi_{i}+\xi_{k} \xi_{i}\right)\right] .
\end{aligned}
$$


Applying $\nabla_{k}$ to $(6.5)$, it follows that

$$
\nabla_{k} H_{j i}^{\prime}=\nabla_{k} \nabla_{j} \varrho_{i}+\nabla_{k} \varrho_{j} \varrho_{i}+\varrho_{j} \nabla_{k} \varrho_{i}-\nabla_{k} \tilde{\varrho}_{j} \tilde{\varrho}_{i}-\tilde{\varrho}_{j} \nabla_{k} \tilde{\varrho}_{i}-\nabla_{k} f_{j i}
$$

from which we get

$$
\begin{aligned}
\nabla_{[k} H_{j] i}^{\prime}=-R_{k j i}{ }^{r} \varrho_{r}+\varrho_{j} \nabla_{k} \varrho_{i}-\varrho_{k i} \nabla_{j} \varrho_{i}-\tilde{\varrho}_{i}\left(\nabla_{k} \tilde{\varrho}_{j}-\nabla_{j} \tilde{\varrho}_{k}\right) & \\
& -\tilde{\varrho}_{j} \nabla_{k} \tilde{\varrho}_{i}+\tilde{\varrho}_{k} \nabla_{j} \tilde{\varrho}_{i}-\nabla_{i k} f_{j] i}
\end{aligned}
$$

and eliminating $\nabla_{\mathrm{I} k} f_{j 3 i}$ from (6.6) and (6.10) and owing to (5.9), (5.11), (6.5) and (6.8), it follows that

$$
\begin{aligned}
& \frac{\beta}{2(n+1)\left(\chi^{2}+\mu^{2}\right)}\left(g_{i j j} \xi_{k]}+\xi_{i j} J_{k] i}+2 \xi_{i} J_{k j}\right) \\
& =Q_{k j i}+\left(-f_{[j}^{\prime}-\varrho_{[j}+b_{[j}+v_{[j}\right) f_{k] i}+\left(f_{[j}^{\prime \prime}-\tilde{\varrho}_{[j}+o_{[j}+w_{[j}\right) f_{k] r} J_{i}{ }^{r}
\end{aligned}
$$

On the other hand, differentiating (6.3) covariantly and regarding to (5.12), (5.14), (6.3) and (6.9), we find

$$
\varkappa_{k} a+x a_{k}=\nabla_{k} \varrho^{\prime}=\nabla_{k} \varrho_{r} \xi^{r}+\varrho^{r} \nabla_{k} \xi_{r}=\varrho_{k}+f_{k}^{\prime}-A \xi_{k}+\varrho^{\prime}\left(b_{k}+v_{k}\right)+\varrho^{\prime \prime}\left(c_{k}+w_{k}\right)
$$

and

$\mu_{k} a+\mu a_{k}=-\nabla_{k} \varrho^{\prime \prime}=-\nabla_{k} \varrho_{r} \xi^{r}-\varrho^{r} \nabla_{k} \xi_{r}=\tilde{\varrho}_{k}-f_{k}^{\prime \prime}+A \xi_{k}-\varrho^{\prime \prime}\left(b_{k}+v_{k}\right)+\varrho^{\prime}\left(c_{k}+w_{k}\right)$,

from which

$$
\varkappa a_{k}=\varrho_{k}+f_{k}^{\prime}-A \xi_{k}+\varrho^{\prime} b_{k}+\varrho^{\prime \prime} c_{k}-v_{k}
$$

and

$$
\mu \tilde{a}_{k}=-\varrho_{k}-f_{k}^{\prime}-A \xi_{k}-\varrho^{\prime \prime} \tilde{b}_{k}+\varrho^{\prime} \tilde{c}_{k}-\tilde{w}_{k}
$$

and consequently, we have

$$
\varkappa a_{k}+\mu a_{k}=-2 A \xi_{k}+\varrho^{\prime}\left(b_{k}+c_{k}\right)+\varrho^{\prime \prime}\left(c_{k}-\tilde{b}_{k}\right)-v_{k}-\tilde{w}_{k},
$$

where we put

$$
A=\frac{(n+2) \alpha+n \beta|\sigma|^{2}}{2(n+1)^{2}}
$$

Now if we put

$$
\left\{\begin{array}{l}
E=\frac{1}{2 \beta|\xi|^{2}}\left[2 \beta|\xi|^{2} A+a\left(x \beta^{\prime}-\mu \beta^{\prime \prime}\right)+2 \beta\left\{x a^{\prime}+a \mu^{2}\left(\nabla_{r} \sigma^{r}-2 n a\right)\right\}\right], \\
F=\frac{1}{2 \beta|\xi|^{2}}\left[a\left(\varkappa \beta^{\prime \prime}+\mu \beta^{\prime}\right)+2 \beta\left\{x a^{\prime \prime}+a \varkappa \mu\left(\nabla_{r} \sigma^{r}-2 n a\right)\right\}\right], \\
G=\frac{1}{2 \beta|\xi|^{2}}\left[a\left(x \beta^{\prime \prime}+\mu \beta^{\prime}\right)+2 \beta\left\{\mu a^{\prime}-a x \mu\left(\nabla_{r} \sigma^{r}-2 n a\right)\right\}\right], \\
H=\frac{1}{2 \beta|\xi|^{2}}\left[-2 \beta|\xi|^{2} A+a\left(\mu \beta^{\prime \prime}-x \beta^{\prime}\right)+2 \beta\left\{\mu a^{\prime \prime}-a x^{2}\left(\nabla_{r} \sigma^{r}-2 n a\right)\right\}\right]
\end{array},\right.
$$


and substitute (6.12) and (6.13) into (6.11), then we have

$$
\begin{aligned}
Q_{k j i}=\frac{\beta}{2(n+1)\left(x^{2}+\mu^{2}\right)}\left(g_{i 1 j} \xi_{k]}+\xi_{[j} J_{k] i}\right. & \left.+2 \xi_{i} J_{k j}\right) \\
& +\left(E \xi_{[k}+F \xi_{[k}\right) f_{j 1 i}+\left(G \xi_{[k}+H \xi_{[k}\right) f_{j] r} J_{i}{ }^{r},
\end{aligned}
$$

because of (5.3), (5.4) and (5.5).

By the way, it follows from (6.15) that

$$
F+G=0
$$

by virtue of (5.7).

In the next place, we shall investigate deep into (5.15). Making use of (6.15), we have

$$
\begin{aligned}
\nabla_{j} \xi_{i}-J_{j}{ }_{j} J_{i} s \nabla_{r} \xi_{s} & =(H-E)\left(\xi_{j} \xi_{i}-\tilde{\xi}_{j} \xi_{i}\right) \\
& =\mathbf{0}
\end{aligned}
$$

which means that

$$
H-E=0
$$

Therefore if we can prove

$$
H+E=0 \text { and } \quad F-G=0,
$$

then we know $E=F=G=H=0$ by virtue of $(6.17)$ and $(6.18)$. In the rest of this section, let us show (6.19).

On the other hand, although M. Matsumoto in [3] has proved that $\nabla^{i} R\left(=g^{i r} \nabla_{r} R\right)$ is contravariant analytic in a compact Kählerian manifold with vanishing Bochner curvature tensor, by means of the equation in the middle of page 27 , we can easily prove this fact without compact. So, in the following we can take account of this fact.

Making use of (4.23) and (4.28), it holds that

$$
\beta_{j}=-2 \beta b_{j},
$$

and we have by means of $(4.26)$, and $(4.32)$ and $(6.20)$

$$
\nabla_{j} R=2(n+1) \alpha_{i} .
$$

Since $\nabla^{j} R$ is contravariant analytic, we get

$$
\nabla_{j} \alpha_{i}=J_{j}{ }^{r} J_{i}{ }^{s} \nabla_{r} \alpha_{s}
$$

To make use of $(6.21)$, let us calculate $\nabla_{j} \alpha_{i}$. We differentiate (4.32) covariantly and use (4.26). Then

$$
\nabla_{j} \alpha_{i}=2\left[\beta a_{i} \sigma_{i}-a \beta b_{j} \sigma_{i}+a^{2} \beta g_{j i}+a \beta c_{j} \tilde{\sigma}_{i}\right],
$$


and substituting this into (6.21) and owing to $(6.20)$, we get

$$
\left(\beta a_{j}-a \beta b_{j}+a \beta \tilde{c}_{j}\right) \sigma_{i}-\left(\beta \tilde{a}_{j}-a \beta \tilde{b}_{j}-a \beta c_{j}\right) \tilde{\sigma}_{i}=0,
$$

which yields that

$$
\beta a_{j}-a \beta b_{j}+a \beta \tilde{c}_{j}=0 .
$$

By virtue of $(5.3),(5.4)$ and $(5.8)$, the equation above becomes

$$
\left\{a^{\prime}-a x\left(\nabla_{r} \sigma^{r}-2 n a\right)\right\} \xi_{j}+\left\{a^{\prime \prime}+a \mu\left(\nabla_{r} \sigma^{r}-2 n a\right)\right\} \xi_{j}=0,
$$

which imply that

$$
a^{\prime}-a x\left(\nabla_{r} \sigma^{r}-2 n a\right)=0, \quad a^{\prime \prime}+a \mu\left(\nabla_{r} \sigma^{r}-2 n a\right)=0
$$

Finally, by virtue of the above equations, it follows from (6.15) that (6.19). Then from (6.16) we have

$$
Q_{k j i}=\frac{\beta}{2(n+1)\left(x^{2}+\mu^{2}\right)}\left(g_{i[j} \xi_{k]}+\xi_{[j} J_{k] i}+2 \xi_{i} J_{k j}\right)
$$

which means that

$$
\nabla_{k} \nabla_{j} z_{i}-\nabla_{j} \nabla_{\bar{k}} z_{i}=-R_{k j i}{ }^{r} z_{r}
$$

making use of (5.9), (6.4) and (6.8) and consequently, the partial differential equations (6.1) are completely integrable under the conditions (I), (II) and (III).

\section{7. - Main theorem.}

Since $(6.1)$ is completely integrable, there exist $2 n$ linearly independent solutions $z_{i}^{\left({ }^{(h)}\right.}$ and any solution is expressible as a linear combination with constant coefficients of these particular solutions. Since $f_{j i}$ and $f_{j r} J_{i}{ }^{r}$ are symmetrie, $z_{i}{ }^{(h)}$ are gradient vectors. Hence if we set

$$
z_{i}^{(k)}=\frac{\partial \bar{x}^{h}}{\partial x^{i}}
$$

then $\bar{x}^{h}=\bar{x}^{h}\left(x^{i}\right)$ are $2 n$ independent functions of $x^{h}$ and consequently may be considered to define a transformation of loeal coordinate system.

Now the Christoffel symbols of the second kind in the coordinate system $\left(\bar{x}^{h}\right)$ have the following transformation low under a coordinate transformation

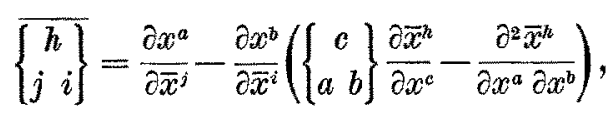


from which we have by (6.1) and (7.1)

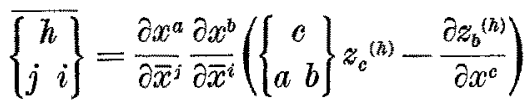

$$
\begin{aligned}
& =-\frac{\partial x^{a}}{\partial \bar{x}^{j}} \frac{x \partial^{b}}{\partial \bar{x}^{i}} \nabla_{a} z_{b}^{(h)} \\
& =\bar{\varrho}_{(j} \delta_{i)}^{h}+\varrho_{(j} \bar{J}_{i)}^{h}+\bar{f}_{j i} \tilde{\bar{\xi}}^{h}-\bar{f}_{j r} \bar{J}_{i}^{r} \tilde{\xi}^{\tilde{\xi}},
\end{aligned}
$$

where $\bar{\varrho}_{i}, \bar{f}_{j i}, \bar{J}_{j}^{h}$ and $\tilde{\bar{\xi}}^{h}$ are components in the local coordinate system $\left(\bar{x}^{h}\right)$ corresponding for $\varrho_{i}, f_{i i}, J_{i}{ }^{h}$ and $\xi^{h}$ respectively.

Finally, we shall show that $M$ is the holomorphically subprojective Kählerian manifold of the first kind under the conditions (I), (II) and (III) stated in $\S 3$. Now we set $z=\xi^{r} z_{r}$. Then we have after some computations

$$
\begin{aligned}
\frac{\partial z}{\partial x^{i}} & =\nabla_{i} z=\nabla_{i} \xi^{r} z_{r}+\xi r \nabla_{i} z_{r} \\
& =z_{i}+E \xi_{i}+F_{i} \\
& =z_{i}
\end{aligned}
$$

by making use of (5.12), (6.1), (6.12), (6.13) and (6.15). Since we have $z^{(h)}=\xi^{r} z_{r}^{(h)}$, it follows that

$$
\frac{\partial z^{(h)}}{\partial x^{i}}=z_{i}^{(h)}=\frac{\partial \bar{x}^{h}}{\partial x^{i}}
$$

from which we get $z^{(h)}=\bar{x}^{h}$ and consequently $\bar{x}^{h}=\xi^{r} z_{r}^{(h)}$, or

$$
\bar{x}^{h}=\xi^{h} .
$$

Taking account of (7.2) and (7.3), we obtain

$$
\left\{\begin{array}{c}
h \\
j i
\end{array}\right\}=\bar{\varrho}_{(j} \delta_{i)}^{h}+\tilde{\bar{\varrho}}_{(j} \bar{J}_{i)}^{h}+\bar{f}_{j i} \bar{x}^{h}-\bar{f}_{j r} \bar{J}_{i} q \tilde{\bar{x}}^{h}
$$

in a suitable local coordinate system $\left(\bar{x}^{h}\right)$. Therefore $M$ is a holomorphically subprojective Kählerian manifold. At last, by Theorem A we can see that $M$ is the holomorphically subprojective Kählerian manifold of the first kind.

Coversely, if $M$ the holomorphically subprojective Kählerian manifold of the first kind, then $M$ has the properties (I), (II) and (III) stated in $\S 3$ by virtue of Theorem A.

Thus we have

MAIN TheoRfur. - In order that a $2 n(n \geqq 3)$ dimensional Kählerian manifold $M$ is the holomorphically subprojective Kählerian manifold of the first kind, it is necessary and sufficient that the following properties (I), (II) and (III) are satisfied in $M$ : 
I) M has a vanishing Bochner curvature tensor which has the Ricci tensor of the form

$$
R_{j i}=\alpha g_{j i}+\beta\left(\sigma_{j} \sigma_{i}+\tilde{\sigma}_{j} \tilde{\sigma}_{i}\right),
$$

where $\sigma_{i}$ is a gradient vector, and $\alpha$ and $\beta$ are some functions.

(I) The vector $\xi^{h}$ given by $\xi^{h}=x \sigma^{h}+\mu \tilde{\sigma}^{h}$ for some functions $x$ and $\mu$ is contravariant analytie.

(III) There exist a gradient vector $\varrho_{i}$ and a symmetric tensor $f_{j i}$ satisfying $f_{r i j} J_{i 1}^{r}=0$ and

$$
\nabla_{k} \varrho_{j}=-\varrho_{k} \varrho_{j}+\tilde{\varrho_{k}} \tilde{\varrho}_{j}+f_{k j}+\phi g_{k j}+\varphi\left(\xi_{k} \xi_{j}+\tilde{\xi}_{k} \tilde{\xi}_{j}\right),
$$

where $\phi$ and $\varphi$ are some functions.

By virtue of (7.2) and the Main Theorem, we have

THEOREM. - The Christoffel symbols of the holomorphically subprojective Kählerian manifold of the first kind take the form

$$
\left\{\begin{array}{c}
h \\
j i
\end{array}\right\}=\varrho_{(j} \delta_{i)}{ }^{h}+\tilde{\varrho}_{(j} J_{i)}{ }^{h}+f_{i i} \xi^{h}-f_{j r} J_{i}{ }^{r} \xi^{h}
$$

for suitable coordinate system $\left(x^{h}\right)$, where $\xi^{\text {h }}$ is a contravariant analytic vector field such that

$$
\nabla_{j} \xi^{h}=\alpha \delta_{j}^{n}+\beta J_{j}^{h}+\gamma_{j} \xi^{n}+\tilde{\gamma}_{j} \tilde{\xi}^{n}, \quad \gamma_{j}=\partial_{j} \gamma .
$$

\section{BIBLIOGRAPHY}

[1] T. Adati, On subprojective spaces I, Tôhoku Math. J., 3 (1953), pp. 159.173.

[2] B. KaGAN, Sur les espaces sous-projectifs, C. R. Acad. Sei. Paris, 191 (1930), pp. 548-550.

[3] M. Matsumoto, On Kahlerian spaces with parallel or vanishing Bochner curvature tensor, Tensor N. S., 20 (1969), pp. 25-28.

[4] T. Otsuki - Y. TastrRo, On ourves in Kählerian spaces, Math. J. Okayama Univ., 4 (1954), pp. 57-78.

[5] P. Rachevsky, Sur les espaces sous-projectifs, C. R. Acad. Sci. Paris, 191 (1930), pp. 547-548.

[6] P. RACHEvsky, Caracteres tensoriels de l'espace sous-projectif, Abh. des Seminars f..r Vektor und Tensoranalysis, Moskau, 1 (1933), pp. 126-140.

[7] S. Tachibaxa, On the Bochner curvature tensor, Nat. Sei. Rep. Ochanomizu Univ., 18 (1967), pp. 15-19.

[8] Y. TAsmro, On holomorphically projective correspondences in an almost complex space, Math. J. Okayama Univ., 6 (1957), pp. 147-152.

[9] S. YaMAGUCHI - T. ADATI, On holomorphically subprojective Kählerian manifolds, I, to appear in Annali di Matematica Pura ed Applicata.

[10] S. YAMAGUCHI - T. ADATI, On holomorphically subprojective Kählerian manifolds, $I I$, to appear.

[11] K. Yano, Differential geometry on complex and almost complex spaces, Pergamon Press (1965).

[12] K. YANo - S. Bochner, Curvature and Betli numbers, Ann. of Math. Stud., 32 (1953).

9 - Annali di Matematica 